\title{
Lobular Neoplasia: Another Reset in Management?
}

\author{
Julie Dunderdale, MD and Seema Ahsan Khan, MD
}

Northwestern Memorial Hospital, Chicago, IL

Lobular neoplasia (LN) has a remarkable history of reinvention, going back to 1955, when Haagensen and colleagues reached a decision to manage women with $\mathrm{LN}$ with observation rather than mastectomy. They reviewed their results on this strategy in 1978, defining the natural history of LN, classifying it as a breast cancer risk factor affecting both breasts, and proposing to change the name of this condition from "lobular carcinoma-in situ" to "lobular neoplasia." ${ }^{1}$ They pointed out that if mastectomy were to be used for this condition, it should rationally be a bilateral procedure, given the bilateral distribution of subsequent cancers. Data generated by Haagensen and his contemporaries guides standards of therapy today, but the term "lobular neoplasia" now also includes atypical lobular hyperplasia which is almost exclusively detected in core needle biopsy samples of screen-detected lesions rather than as an incidental microscopic diagnosis in excisions of palpable lumps. Advances in imaging are leading to increased diagnosis of this problem, which is found at a frequency of about 0.2 per 1000 film-screen mammograms, and 0.6 per 1000 digital mammograms, a threefold increase with the use of digital mammography. ${ }^{2}$

Present uncertainties regarding LN include subjective variation between pathologists in the diagnosis of atypical lobular hyperplasia versus lobular carcinoma-in situ; the recent reemergence of $\mathrm{LN}$ as a precursor rather than a risk marker in some settings, and, importantly for surgeons, the frequency with which underdiagnosis of invasive cancer or ductal carcinoma-in situ (DCIS) occurs when LN is detected using core needle biopsy. ${ }^{3}$ A low frequency of underdiagnosis implies that surgical excision does not have to follow a core biopsy diagnosis (as National

(C) Society of Surgical Oncology 2015

First Received: 2 October 2015; Published Online: 16 December 2015

S. A. Khan, MD

e-mail: skhan@nmh.org; s-khan2@northwestern.edu
Comprehensive Cancer Network guidelines currently recommend for lobular carcinoma-in situ). ${ }^{4}$ However, if the frequency is high, follow-up surgical excision to rule out cancer would be wise. A large retrospective literature on this aspect of $\mathrm{LN}$ has recently been converging to suggest that the frequency of cancer is low in the presence of good radiologic-pathologic concordance, and observation without excision is acceptable. ${ }^{5,6}$ In this issue of Annals of Surgical Oncology, Nakhlis et al. add a nice prospective study to this literature; they assess the upgrade rate of LN diagnosed at core biopsy, and they find it to be low enough to warrant a recommendation against routine excision of these lesions. Their study has several significant strengths, including the multi-institutional setting, prospectively defined criteria for inclusion, a formal statistical plan, and central review of the pathologic material. However, in terms of the adoption of this recommendation by the broad community of practitioners in this country and elsewhere, the devil remains firmly ensconced in the details.

Nakhlis et al. had a primary objective of defining the frequency of upgrades, and they assumed a priori that a difference in upgrade rate of 5 vs. $15 \%$ would be meaningful; therefore, they estimated that if 7 (9\%) of 78 or fewer subjects experienced an upgrade, the true rate was likely to be $5 \%$ at type I error less than $5 \%$, and the study could be halted. This approach, although reasonable for the specific goal of establishing the upgrade rate with confidence, does leave open the question of how well their 77 participants represent the entire population of women with LN. For example, there are only 16 women with a mammographic density, and no description of ultrasound findings was provided. Because the presence of a density (on mammogram or ultrasound) has been associated with a higher probability of upgrade, the small number of subjects presenting with a density leaves some uncertainty as to the applicability of the data from this study to this particular subset. $^{7}$ Another aspect is the microscopic extent of the lesion. Existing data suggest that the involvement of more 
than 3 terminal duct lobular units with LN is a predictor of a higher upgrade rate, but with only two upgrades in their study, Nakhlis et al. cannot report on these aspects, and it is not possible to place their data into the context of the available literature. ${ }^{8}$ Thus the "tyranny of $\mathrm{N}$ " remains because the tiny number of events limits the conclusions that can be drawn.

The thorniest problem in the management of women with screen-detected, core biopsy-proven $\mathrm{LN}$ is that of radiologic-pathologic concordance. Despite general agreement that such concordance is the key to management without excision, the precise definition of concordance remains somewhat mysterious in their data and in that of others. Concordance is generally taken to mean that the radiologic appearance of a lesion matches the pathologic appearance; the implication is straightforward for lesions where a classical radiologic appearance is widely accepted (pleomorphic calcifications for DCIS, spiculated densities for invasive cancer), but when there is no classical radiologic morphology to a given pathologic finding, the definition of concordance becomes trickier. Nakhlis et al. declare at the outset that $\mathrm{LN}$ lacks a distinct radiologic correlate; concordance then relies on the presence (at the microscopic level) of an accompanying lesion that explains the calcifications, density, or other finding that led to the core biopsy. For example, calcifications are seen in areas of adjacent usual ductal hyperplasia or sclerosing adenosis; or a fibroadenoma explains a radiologic mass. Thus, the radiologic and pathologic findings agree that the $\mathrm{LN}$ is incidental and does not require excision. The application of this rule of concordance to clinical practice requires a specific statement regarding concordance of findings in the final radiology report describing the results of the biopsy; surgeons must therefore seek this clarification, if not explicitly stated, before counseling their patients that a given lesion does not require excision. A final point relates to the role of postbiopsy imaging because calcifications are often ablated by the core biopsy procedure. Should the presence of residual calcifications be factored into decision making? Again, turning to the literature, it appears that concordance is the key; if a good (benign) explanation for the index radiologic finding is present in the core biopsy, then the presence of a residual radiographic finding is tolerable but must be followed. ${ }^{8}$

Overall, then, this study validates what larger retrospective analyses have previously shown: that there is a low risk of upgrading classical $\mathrm{LN}$ found on core needle biopsy to DCIS or invasive cancer in the specific setting of nonpalpable, mammographically detected lesions with concordance between radiology and pathology findings, as determined by an experienced multidisciplinary team. It is notable in this regard that the majority of the patients enrolled by Nakhlis et al. came from two institutions (the comprehensive cancer centers of Dana Farber/Harvard and Memorial Sloan Kettering), whereas four other high-volume tertiary care centers contributed a total of eight patients, raising questions about selectivity of enrollment at these other institutions.

In conclusion, Nakhlis and colleagues report a small and narrowly focused study, which is nevertheless clean in design and conclusions. In the messier world at large, its caveats must be remembered, and patient selection for observation must be as careful as that described here. For the future, a larger prospective trial is needed-one that is more inclusive and allows examination of both the predictors of cancers that accompany LN and those that occur subsequently, along with their biologic spectrum. Recent data suggest that these may be fairly indolent, thereby allowing greater latitude to the strategy of observation of these lesions, particularly when accompanied by medical therapy for prevention. ${ }^{9}$ We may then be looking at a model that allows management of LN without excision for the majority of women with this breast lesion.

DISCLOSURE The authors declare no conflict of interest.

\section{REFERENCES}

1. Haagensen CD, Lane N, Lattes R, Bodian C. Lobular neoplasia (so-called lobular carcinoma in situ) of the breast. Cancer. 1978;42:737-69.

2. Neal CH, Coletti MC, Joe A, Jeffries DO, Helvie MA. Does digital mammography increase detection of high-risk breast lesions presenting as calcifications? AJR Am J Roentgenol. 2013;201: $1148-54$.

3. King TA, Reis-Filho JS. Lobular neoplasia. Surg Oncol Clin N Am. 2014;23:487-503.

4. Theriault RL, Carlson RW, Allred C, et al. Breast cancer, version 3.2013: featured updates to the NCCN guidelines. J Natl Compr Canc Netw. 2013;11:753-60.

5. Shah-Khan MG, Geiger XJ, Reynolds C, Jakub JW, Deperi ER, Glazebrook KN. Long-term follow-up of lobular neoplasia (atypical lobular hyperplasia/lobular carcinoma in situ) diagnosed on core needle biopsy. Ann Surg Oncol. 2012;19:3131-8.

6. Zhao C, Desouki MM, Florea A, Mohammed K, Li X, Dabbs D. Pathologic findings of follow-up surgical excision for lobular neoplasia on breast core biopsy performed for calcification. Am J Clin Pathol. 2012;138:72-8.

7. Brem RF, Lechner MC, Jackman RJ, et al. Lobular neoplasia at percutaneous breast biopsy: variables associated with carcinoma at surgical excision. AJR Am J Roentgenol. 2008;190:637-41.

8. Middleton LP, Sneige N, Coyne R, et al. Most lobular carcinoma in situ and atypical lobular hyperplasia diagnosed on core needle biopsy can be managed clinically with radiologic follow-up in a multidisciplinary setting. Cancer Med. 2014;3:492-9.

9. Abdel-Fatah TM, Powe DG, Hodi Z, Reis-Filho JS, Lee AH, Ellis IO. Morphologic and molecular evolutionary pathways of low nuclear grade invasive breast cancers and their putative precursor lesions: further evidence to support the concept of low nuclear grade breast neoplasia family. Am J Surg Pathol. 2008;32:513-23. 